\title{
Growth and characterisation of triammonium citrate
}

\author{
$M$ VENKATESHWARLU $\dagger, T$ BHASKAR RAO* and K KISHAN \\ RAO \\ Department of Physics, Kakatiya University, Warangal 506009 , India \\ †Department of Physics, CKM Arts and Science College, Warangal 506 006, India \\ MS received 21 October 1988; revised 12 January 1989
}

\begin{abstract}
Single crystals of triammonium citrate are grown from aqueous solution and characterized using $\mathrm{X}$-ray and etching studies. Using single crystal $\mathrm{X}$-ray diffraction, the crystal is found to be orthorhombic with lattice parameters $a=6.223 \AA, b=15.048 \AA$ and $c=11.056 \AA$. Surface studies on the as-grown faces reveal stratigraphic patterns. Of the several etchants tried, glacial acetic acid appears to be the best etchant for revealing dislocation sites on the as-grown faces.
\end{abstract}

Keywords. Triammonium citrate; lattice parameters; etching; stratigraphic pattern.

\section{Introduction}

Citrates are important compounds having biological and medical applications (Lonerdal et al 1980; Swanson et al 1983). Till now no systematic study of their crystal growth and characterization has been reported. The crystal structures of many of these compounds are not known and neither are their morphological features. A study of the citrates is undertaken in the laboratory.

In this paper we report the growth and characterization of triammonium citrate $\left[\left(\mathrm{NH}_{4}\right)_{3} \mathrm{C}_{6} \mathrm{H}_{5} \mathrm{O}_{7}\right]$ using $\mathrm{X}$-ray and etching techniques.

\section{Results and discussion}

\subsection{Crystal growth}

Single crystals of triammonium citrate are grown from saturated aqueous solution by slow evaporation at room temperature. The morphology of single crystals obtained is shown in figure 1. It can be seen that there are two predominant faces, one in the form of an elongated hexagon ( $c$ face) and the other in the form of a rectangle ( $b$ face). These two faces are perpendicular to each other.

\section{$2.2 X$-ray studies}

As the morphology and crystal structure are not known, Laue photographs of faces $c$ and $b$ are taken (figure $2 \mathrm{a}, \mathrm{b}$ ). Face $c$ (figure 2a) exhibits a $C_{2 v}$ symmetry whereas the other (figure $2 \mathrm{~b}$ ) shows a $C_{s}$ symmetry. This symmetry suggests that the crystal must have the minimum symmetry of an orthorhombic crystal.

Further, to confirm the above results, rotation photographs of the crystal about

\footnotetext{
${ }^{*}$ For correspondence.
} 


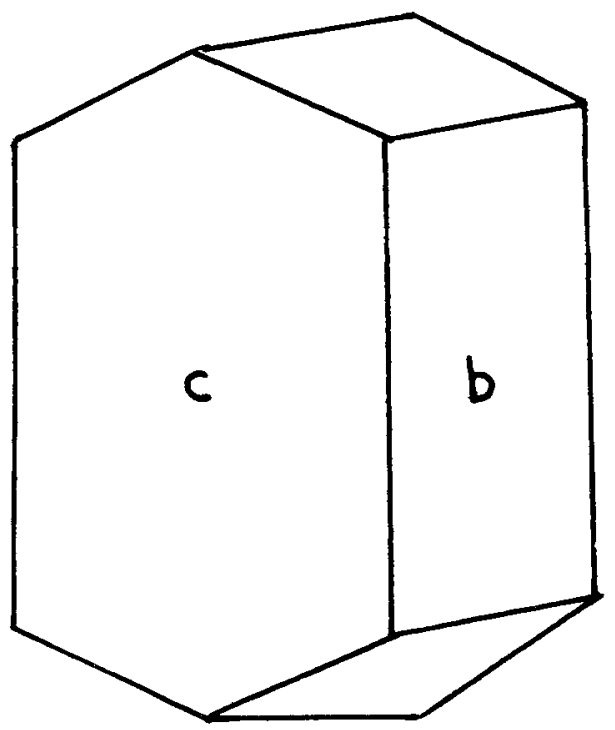

Figure 1. Morphology of triammonium citrate crystal ( $c-a b$ plane, $b-a c$ plane).

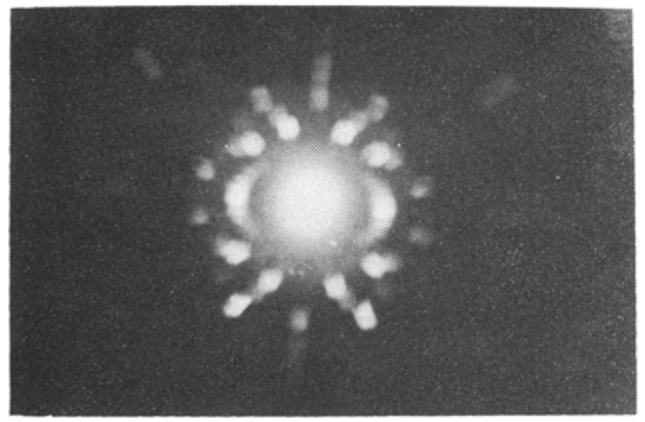

(a)

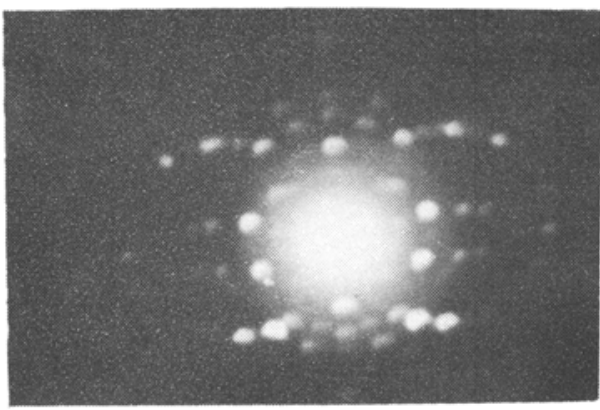

(b)

Figure 2. Laue photograph of triammonium citrate, (a) hexagonal face ( $a b$ plane), (b) rectangular face (ac plane).

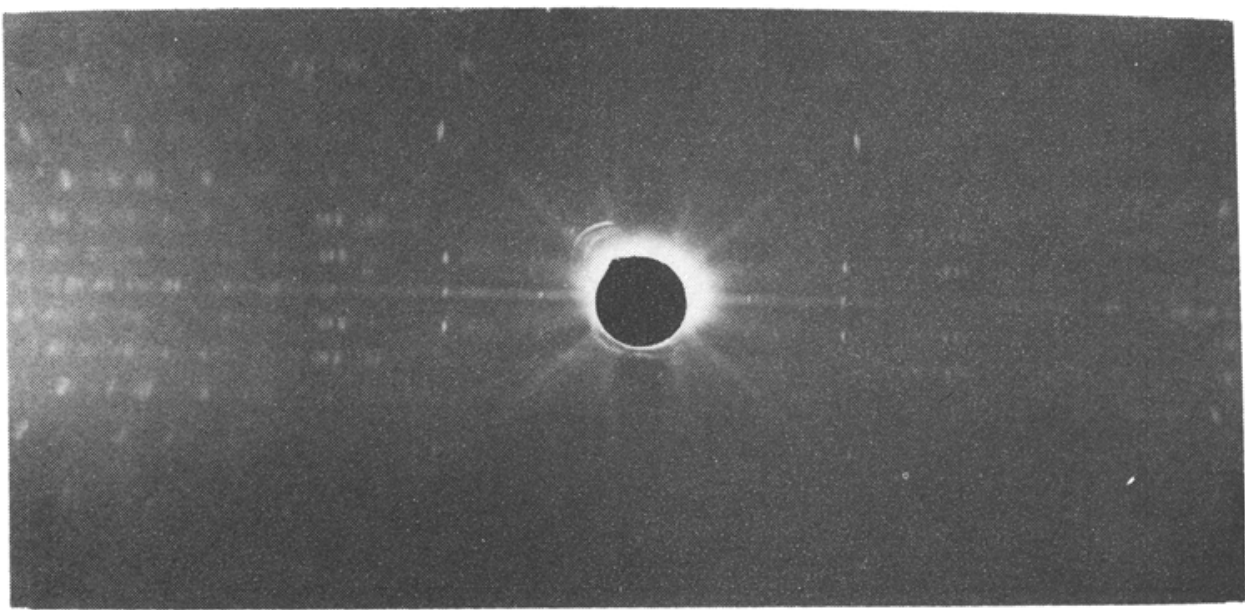

Figure 3. Rotational photograph around the $b$ axis. 
the axes perpendicular to faces $c$ and $b$, and the axis perpendicular to these two axes are taken. Figure 3 shows one of the rotation photographs. It has been found that all the photographs taken about the above mentioned axes show layered lines confirming that the crystal is orthorhombic. The prominent spots in all photographs are indexed and the unit cell dimensions calculated are $a=6 \cdot 223 \AA$, $b=15.048 \AA$ and $c=11.056 \AA$. Thus, the $c$ face is the $a b$ plane and the $b$ face the $a c$ plane.

\subsection{Selective etching studies}

Various etchants have been tried on all the faces of these crystals and glacial acetic acid is found to be the best etchant on the ac plane. Apart from this, formic acid

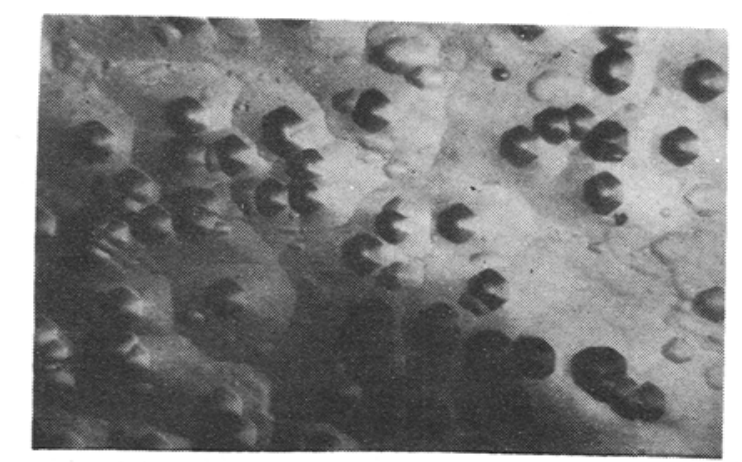

(a)

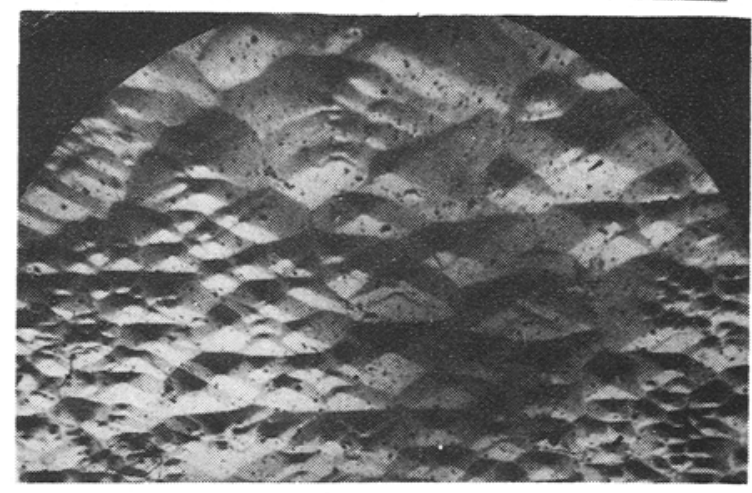

(b)

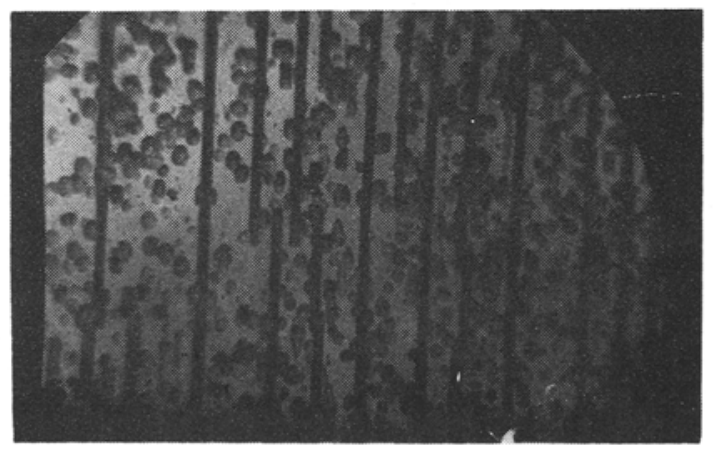

(c)

Figure 4. Etch pit pattern on ac face of triammonium citrate, (a) etched with acetic acid $(\times 120)$, (b) etched with water $(\times 100)$, (c) Stratigraphic pattern $(\times 100)$. 
and water also have some etching action on this face. However, all these etchants do not have any etching action on the other faces. Figure 4a shows the etch pattern obtained on etching with glacial acetic acid for $20 \mathrm{~s}$. The etch figures are hexagonal in shape. To establish the selective action, repeated etching studies have been made. It is observed that no new etch pits are formed and an increase in their size indicates the formation of pits at dislocation sites. Figure $4 \mathrm{~b}$ shows the etch pattern obtained with water.

Some of the crystals reveal some lines on the as-grown rectangular faces, parallel to one of the edges but perpendicular to the hexagonal faces. Thus, these lines have a definite crystallographic orientation parallel to the $c$-axis of the crystal. These stratigraphic patterns develop on etching as shown in figure 4c. The pattern persists on repeated etching, thus showing that it runs throughout the body. It suggests that crystal growth of triammonium citrate takes place through successive sheet layer formation (Patel and Desai 1965).

\section{Acknowledgements}

The authors thank Prof. Dinker Sir Deshmukh for useful discussions. One of the authors (MV) acknowledges the University Grants Commission, New Delhi, for financial assistance.

\section{References}

Lonerdal B, Stanislowski A C and Hurley L S 1980 J. Inorg. Biochem. 1271

Patel A R and Desai C C 1965 Acta Crystallogr. 18373

Swanson R, Ilsley W H and Stanislowski A C 1983 J. Inorg. Biochem. 18187 